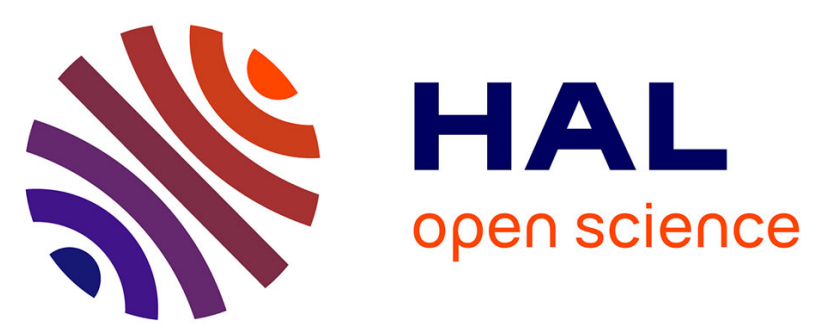

\title{
Validation of a novel luminal flow velocimeter with video fluoroscopy and manometry in the human esophagus
}

J.M. Andrews, H. Nathan, Charles-Henri Malbert, M.A.M.T. Verhagen, M. Gabb, G.S. Hebbard, D. Kilpatrick, S. Macdonald, C.K. Rayner, S. Doran, et al.

\section{To cite this version:}

J.M. Andrews, H. Nathan, Charles-Henri Malbert, M.A.M.T. Verhagen, M. Gabb, et al.. Validation of a novel luminal flow velocimeter with video fluoroscopy and manometry in the human esophagus. AJP - Gastrointestinal and Liver Physiology, 1999, 276 (4), pp.G886-G894. hal-02697049

\section{HAL Id: hal-02697049 \\ https://hal.inrae.fr/hal-02697049}

Submitted on 1 Jun 2020

HAL is a multi-disciplinary open access archive for the deposit and dissemination of scientific research documents, whether they are published or not. The documents may come from teaching and research institutions in France or abroad, or from public or private research centers.
L'archive ouverte pluridisciplinaire HAL, est destinée au dépôt et à la diffusion de documents scientifiques de niveau recherche, publiés ou non, émanant des établissements d'enseignement et de recherche français ou étrangers, des laboratoires publics ou privés. 


\section{J. M. Andrews, H. Nathan, C. H. Malbert, M. A. M. T. Verhagen, M. Gabb, G. S. Hebbard, D. Kilpatrick, S. MacDonald, C. K. Rayner, S. Doran, T. Omari, E. O'Young, C. Frisby, R. J. Fraser, M. Schoeman, M. Horowitz and J. Dent Am J Physiol Gastrointest Liver Physiol 276:886-894, 1999.}

You might find this additional information useful...

This article cites 9 articles, 1 of which you can access free at: http://ajpgi.physiology.org/cgi/content/full/276/4/G886\#BIBL

This article has been cited by 1 other HighWire hosted article:

Nutrient-induced spatial patterning of human duodenal motor function J. M. Andrews, S. M. Doran, G. S. Hebbard, C. H. Malbert, M. Horowitz and J. Dent Am J Physiol Gastrointest Liver Physiol, March 1, 2001; 280 (3): G501-G509. [Abstract] [Full Text] [PDF]

Medline items on this article's topics can be found at http://highwire.stanford.edu/lists/artbytopic.dtl on the following topics:

Physiology .. Esophagus

Medicine .. Fluoroscopy

Physiology .. Humans

Updated information and services including high-resolution figures, can be found at: http://ajpgi.physiology.org/cgi/content/full/276/4/G886

Additional material and information about AJP - Gastrointestinal and Liver Physiology can be found at: http://www.the-aps.org/publications/ajpgi

This information is current as of September 6, 2010 . 


\title{
Validation of a novel luminal flow velocimeter with video fluoroscopy and manometry in the human esophagus
}

\author{
J . M. ANDREWS, ${ }^{1}$ H. NATHAN ${ }^{2}$ C. H. MALBERT, ${ }^{3}$ M. A. M. T. VERHAGEN ${ }^{4}$ M. GABB,${ }^{5}$ \\ G. S. HEBBARD, ${ }^{1}$ D. KILPATRICK,${ }^{6}$ S. MACDONALD ${ }^{2}$ C. K. RAYNER, ${ }^{1}$ S. DORAN,${ }^{1}$ T. OMARI, \\ E. OYOUNG, ${ }^{2}$ C. FRISBY, ${ }^{2}$ R. J . FRASER, ${ }^{1}$ M. SCHOEMAN, ${ }^{2}$ M. HOROWITZ, ${ }^{1}$ AND J . DENT ${ }^{2}$ \\ Departments of ${ }^{1}$ Medicine, ${ }^{2}$ Gastrointestinal Medicine, and ${ }^{5}$ Radiology, Royal Adelaide Hospital, \\ and 'Department of Gastroenterology, Women's and Children's Hospital, Adelaide, South Australia \\ 5000; ${ }^{6}$ Department of Cardiology, Royal Hobart Hospital, Hobart, Tasmania 7000, Australia; \\ 3Station de Reserches Porcines, Institut National de la Recherche Agronomique, Saint-Gilles 35590, \\ France; and ${ }^{4}$ Gastrointestinal Motility U nit, University Hospital, Utrecht 3508, The Netherlands
}

\begin{abstract}
Andrews, J . M., H. Nathan, C. H. Malbert, M. A. M. T. Verhagen, M. Gabb, G. S. Hebbard, D. Kilpatrick, S. MacDonald, C. K. Rayner, S. Doran, T. Omari, E. O'Young, C. Frisby, R. J. Fraser, M. Schoeman, M. Horowitz, and J . Dent. Validation of a novel luminal flow velocimeter with video fluoroscopy and manometry in the human esophagus. Am. J . Physiol. 276 (Gastrointest. Liver Physi ol . 39): G886-G894, 1999.-There is currently no ideal method for concurrently assessing intraluminal pressures and flows in humans with high temporal resolution. We have devel oped and assessed the performance of a novel fiber-optic laser-Doppler velocimeter, mounted in a multichannel manometric assembly. Vel ocimeter recordings were compared with concurrent fluoroscopy and manometry following 50 barium swallows in healthy subjects. During these swallows, the velocimeter sensor was situated in either the proximal (24 swallows) or the distal (26 swallows) esophagus. It signaled intraluminal flow following 46 of 50 swallows. A greater mean number of deflections were recorded in the distal compared with the proximal esophagus (4.3 vs. $2.4, \mathrm{P}=0.001)$. The maximal flow velocity recorded did not differ between the proximal and distal esophagus (76.7 vs. $73.8 \mathrm{~mm} / \mathrm{s}$ ). No vel oci meter signals commenced after fluor oscopic lumen occlusion. The vel ocimeter signals were closely temporally related to fluoroscopic barium flow. U pward catheter movement on swallowing sometimes appeared to cause a velocimeter signal. Manometrically "normal" swallows were no different from "abnormal" swallows in the number and velocity of deflections recorded by the velocimeter. This novel instrument measures intraluminal flow velocity and pressures concurrently, thus enabling direct study of pressure-flow relationships. Flow patterns differed between the proximal and distal esophagus.
\end{abstract}

intraluminal flow; pressure-flow relationships; laser-Doppler flow measurement

THE OVERALL ABORAL direction of flow within the gut is the net result of individual episodes of flow that occur in both directions (3). Relatively little is known about these patterns of flow and the patterns of luminal pressures that cause them. We are currently able to monitor intraluminal pressures with a spatial resolution as close as $1 \mathrm{~mm}$ between each of up to 21 recording

The costs of publication of this article were defrayed in part by the payment of page charges. The article must therefore be hereby marked "advertisement" in accordance with 18 U.S.C. Section 1734 solely to indicate this fact. sideholes. Exact temporal resolution of these pressures is also possible due to the capacity of computer-based recording systems for high-frequency data acquisition. Concurrent recordings of intraluminal flow with a similar temporal resolution are essential for determining how these individual pressure events relate to luminal flow. Previously, it has not been possible to monitor pressures and intraluminal flows simultaneously in humans with a temporal resolution of $<1 \mathrm{~s}$, although suitable methods for use in animals exist (8). The methods that are currently available for evaluating luminal flows in humans include radiographic contrast studies (5), Doppler ultrasonography (1), radiographic marker studies (4), scintigraphy (11), and impedance plethysmography (12). These techniques have substantial limitations with respect to the length of observation that is permissible, reliability of the measurement, and/or the spatial-temporal resolution possible.

We have recently devel oped a laser-Doppler vel ocimeter suitable for use in the gut lumen that measures the polarity and vel ocity of liquid movement, with a temporal resolution of between 4 and $7 \mathrm{~Hz}$ (10). This device was embedded in a multilumen silicone rubber manometric assembly (Dentsleeve, Wayville, SA, Australia) to allow concurrent measurement of intraluminal pressures. The potential advantages of this device include complete el ectrical insulation, high frequency detection of flows, and the absence of radiation exposure.

In this study, we aimed to 1) perform safety and bench testing of the instrument, 2) validate the laserDoppler signal in vivo as an indicator of flow by comparing the velocimeter signal with fluoroscopically observed flow in the human esophagus, 3) define more precisely the patterns of esophageal luminal flows during swallowing, and 4) if possible, determine the relationships between intraluminal flows and pressures.

We chose to perform these studies in the esophagus because of the ease of triggering episodes of bolus flow predictably and the ability to observe them fluoroscopically. We report here the analyses of esophageal luminal pressures and flows that occurred during 50 barium swallows recorded with concurrent video barium fluoroscopy, laser-Doppler velocimeter, and a water-perfused multichannel manometric device. 
Master computer:pressures \& velocity recorded on-line signal processing computer
Avalanche photodiode
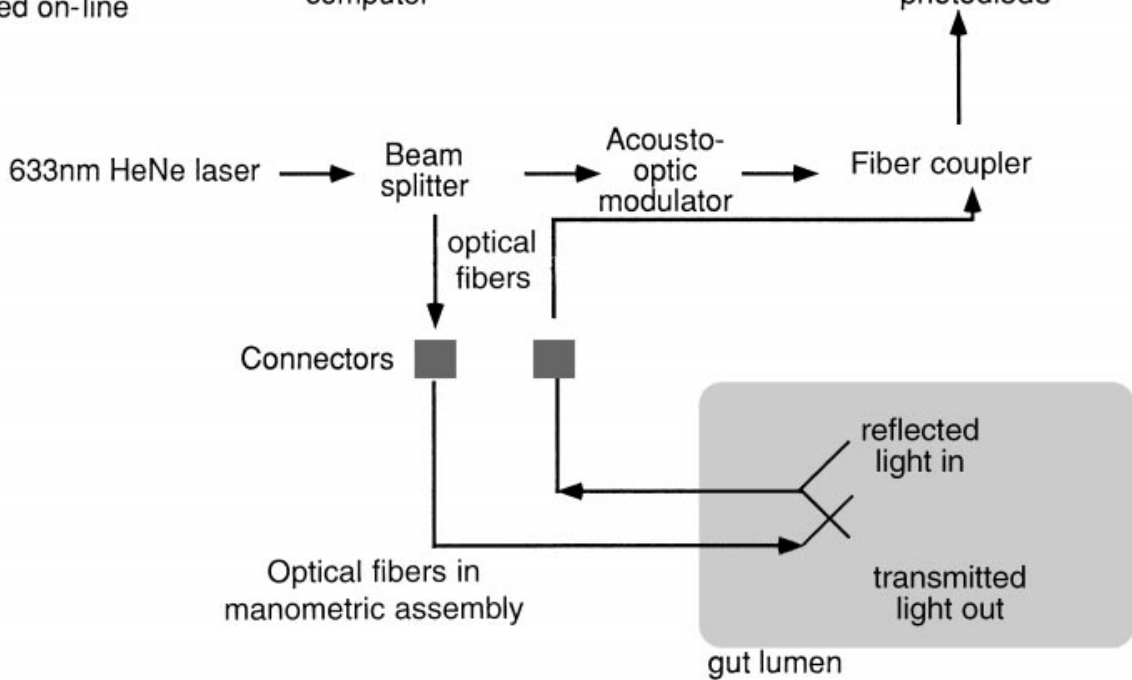

Fig. 1. Schematic of the laser-Doppler velocimeter. For detailed description, see text.

\section{METHODS}

\section{Design of theVel ocimeter System}

Design and system overview. A schematic of the equipment is illustrated in Fig. 1. Light from a helium-neon laser (633 $\mathrm{nm}$ ) is split into two beams, with half the beam transmitted down an optical fiber that has its distal end embedded in a manometric assembly. The optical fiber has an external diameter of $125 \mu \mathrm{m}$; however, the transmitted light is unimodal because it is restricted to a $4 \mu \mathrm{m}$ core within the center of the fiber. The transmitted light enters the gut lumen and is reflected and Doppler-shifted by the passing particles it encounters, according to the Doppler principle. Some of this reflected light is recaptured by a second optical fiber within the assembly, which transmits it back to a photodiode in which the frequency of the reflected light is compared with that of the original beam.

Direction of flow is determined by beating the reference beam with a pulse of known frequency $(+80 \mathrm{MHz})$ from an acousto-optic modulator so that the direction of frequency shifts of the reflected light is evident. This frequency shift is processed, initially in a spectrum analyzer (a specially modified and screened UHFNHF spectrum analyzer, no. K 7620, Dick Smith) and then on-line by a computer (Macintosh II ci,
Apple, Cupertino, CA) using Labview 3.1.1 (National Instruments, Austin, TX)-based computer analysis software that was custom-written in-house (by Mal bert). Thesignal processing software converts the observed Doppler shift into both a velocity and direction of flow.

The system was devel oped to detect vel ocities ranging from 30 to $120 \mathrm{~mm} / \mathrm{s}$. The characteristics of the components limited the vel ocity sampling frequency to $4-7 \mathrm{~Hz}$. The velocity signal was logged concurrently with the manometric data on a second computer (Power Macintosh 7100/80, Apple). The signal processing gave an $\sim 0.4-5$ internal delay from the actual velocity measurement to logging; this was corrected beforeanalysis.

Laser sensor tip. Two separate sensor tips were used: 1) a simple design was used for the bench calibration, consisting merely of the two optical fibers held at a fixed angle $\left(\sim 3^{\circ}\right)$ to each other with adhesive, and 2) for the concurrent manometry and velocimetry, the optical fibers were incorporated into a multichannel manometric assembly (F ig. 2). The fibers were passed down the shaft of the extrusion within individual channels until they reached the last few millimeters of their course. At that point, they entered a common space, formed by drilling a hole perpendicular to the side of the extrusion. An acrylic plug was molded around the two optical fibers, which

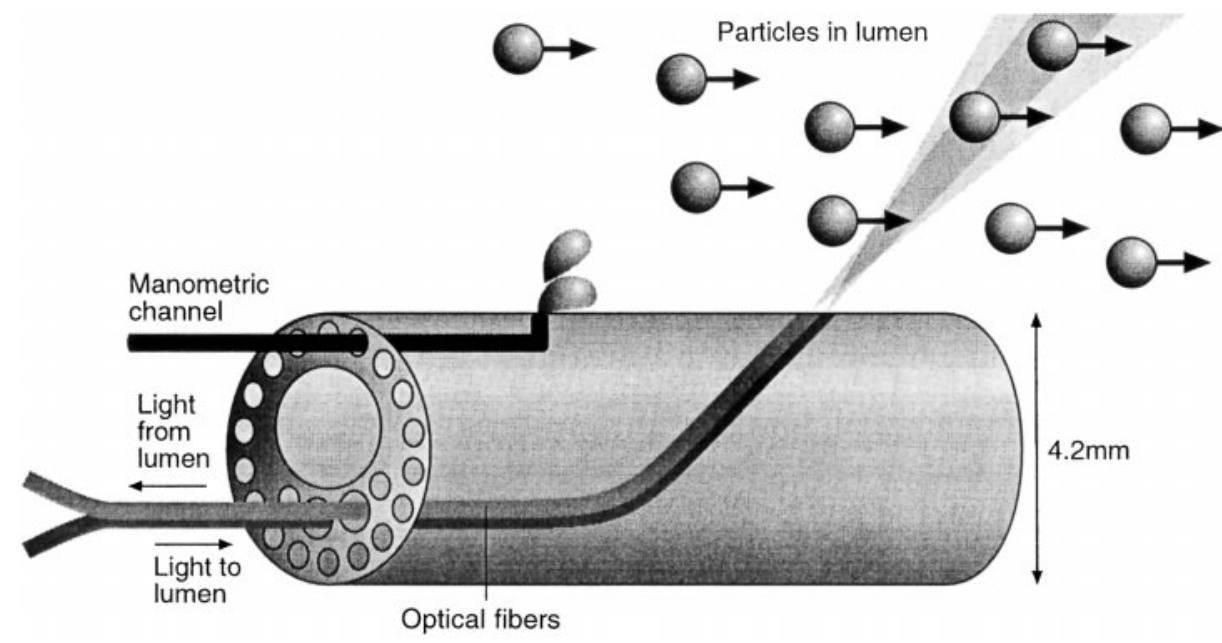

Fig. 2. Schematic of the manometricvelocimetric assembly, illustrating the arrangement of the optical fibers within the assembly and the area over which velocity measurements are made. See text for further detail. 
were held in correct position by a jig. The plug with the embedded optical fibers was then glued into the trephined hole in the manometric assembly. The optical fibers curved toward the side of the extrusion at an angle of $30^{\circ}$ and were angled relativeto each other $\left(\sim 3^{\circ}\right)$ so that their light samplingprojecting areas overlapped 1-2 $\mathrm{mm}$ from the side of the assembly (see Fig. 2). This area of overlap determined the zone of luminal contents within which velocity measurements were made. During the molding process, the luminal ends of the fibers were made to protrude $\sim 1 \mathrm{~mm}$ from the side wall of the assembly. The tips of the fibers were encased in a mound of epoxy glue. This mound of epoxy was ground down with the ends of the optical fibers to optimize the focus and overlap of the fibers' respective cones of projection. This process was assessed visually by evaluating the fibers' cones of projection and directly by verifying the sensor's ability to detect flow while it was manufactured.

Software Information from the spectrum analyzer was processed on-line. The software's major functions were to 1) remove the residual spectral component of the incident laser light, 2) detect the Doppler shift, and 3) calculate the mean velocity.

Briefly, the software acquired in burst the full spectrum supplied by the analog spectrum analyzer, processed all the information comprised in each data set, and, finally, sent the computed vel ocity to a digital-to-anal og converter. Each sweep of the spectrum analyzer, which took $5 \mathrm{~ms}$, was digitized at 5 $\mathrm{kHz}$ with a spectral resolution of $10 \mathrm{~Hz}$. This set of data was then subtracted, point by point, from a similar set acquired with zero velocity (null template). Because, in the absence of fluid motion, the only spectral component is the residual spectral component of the incident laser light, the digital subtraction resulted in the removal of this residual spectral component present in the original signal. A shift in the center frequency of the residual spectral component was corrected for by recalculation of the null template every $120 \mathrm{~s}$. The relevance of this repeated subtraction procedure becomes apparent when the relatively infrequent occurrence of intraluminal flow is compared with no flow. At the pyloric level, for example, flow episodes last for only a brief period of each contractile cycle (9). Without this continual correction of the null signal, it would not be possible to distinguish flow episodes from shift in the center frequency over time.

When the signal had been processed to the point at which the only frequency component present was the Dopplershifted frequency, the software identified the position of the maximal and minimal values of thesignal. A linear regression was constructed using all the data points present between these two extremes; the midvalue of this regression line represented the actual mean velocity. Once converted from hertz to meters per second, this value was presented to the digital to analog converter and a new acquisition from the spectrum analyzer commenced.

\section{Bench Testing: Turntable Validation}

A circular disk with three circumferential grooves cut into it at varying distances from the center was mounted on a turntable and rotated at set speeds, generating movements of known velocities in liquid (dilute milk or lipid emulsion) within the grooves relative to the fixed laser sensor. The number of revolutions of the disk was verified by marking a point on its perimeter and counting revolutions within a fixed time interval. The velocity of the movement of the liquid in each groove was thus calculated. The laser sensor tip was held static in a jig and submerged in the center of the liquid, while the disk was rotated, and the laser-Doppler velocimeter signal was recorded. This recorded velocity was then com- pared with the calculated velocity of the liquid. Measurements were made after the fluid had been rotated for 1-2 min so that the velocity of the liquid within the groove was equal to that of the groove itself.

\section{Safety Assessments}

Calculations. The energy output of the laser is $15 \mathrm{~mW}$ (in free space); however, due to the beam splitter and coupling losses, the maximum power that can be emitted at the fiber tip is $1.5 \mathrm{~mW}$ (given the beam diameter of $4 \mu \mathrm{m}$ ). With regard to eye safety, the beat was focused at a distance of $100 \mathrm{~mm}$ from the eye (the beam cannot be focused any closer); at this distance, the beam diameter spreads out to $15 \mathrm{~mm}$ and the intensity of energy falls well within the limit of $25 \mathrm{~W} / \mathrm{m}^{2}$ for a class 3A laser product (13). With regard to the likelihood of tissue burn, the maximum permitted exposure, under this standard, for a duration of $10 \mathrm{~s}$ or more is $2 \mathrm{~kW} / \mathrm{m}^{2}$. If direct (continuous) tissue contact with the sensor tip is assumed, the velocimeter delivers energy with a calculated intensity of $120 \mathrm{~kW} / \mathrm{m}^{2}$. However, at a distance of only $3.25 \mathrm{~mm}$ in air or 6 $\mathrm{mm}$ in water from the sensor tip, the intensity falls under the $2 \mathrm{~kW} / \mathrm{m}^{2}$ limit. It should be emphasized that two other factors mitigate against the likelihood of tissue burn with prolonged use of the velocimeter: 1) the known ability of tissues and local blood flow to act as a heat sink and 2) the narrowness of the 4- $\mu \mathrm{m}$ beam, which makes it highly unlikely that it will remain in direct contact with the exact same patch of tissue for any substantial period of time, in a living organism, given the mobility of tissue.

Direct tissueassessment. Because of the theoretical possibility of tissue burns resulting from use of the velocimeter, the process was evaluated in vivo in anesthetized rats, according to a protocol approved by theAnimal Ethics Committee of the Institute of Medical and Veterinary Science (Adelaide). The sensor tip was inserted via a gastrotomy and placed in contact with the proximal intestinal mucosa for varying periods (30, 60 , and $120 \mathrm{~min}$ ). Between one and three observations were made per animal (four animals were used). The sensor was held stationary by use of a jig, and its position could be clearly recognized by the light visible through the intestinal wall. The position of the light was marked by a suture on the serosal surface. Threeanimals werekilled immediatel y following the experiment, yielding two observations for each time period. A further animal was all owed to recover for 3 days and then killed. Tissue was examined macroscopically, and three circumferential sections at 2- to 5-mm intervals were taken at each level marked by the sutures. Sections were fixed and stained with hematoxylin and eosin before light microscopy, and histological examination was performed by a qualified pathologist.

\section{Human Study}

Subjects. Eight healthy subjects ( 6 males, 2 females) from 19 to $39 \mathrm{yr}$ old were recruited by advertisement. The subjects had no upper gastrointestinal symptoms and were not taking any regular medication.

Protocol. The study was approved by the Human Research Ethics Committee of the Royal Adelaide Hospital, and all subjects gave written informed consent.

The subjects reported to the Radiol ogy Department after a 6-h fast. The recording assembly was introduced transnasally after local anesthetic spray had been applied. The volunteers then stood upright in front of the fluoroscopy tube, and the velocimeter sensor was positioned according to the pressure patterns recorded by the array of sideholes that straddled it. Initially, the sensor was positioned $\sim 5 \mathrm{~cm}$ above the lower 
esophageal sphincter and later withdrawn to lie $\sim 5 \mathrm{~cm}$ bel ow the upper esophageal sphincter. With the assembly in each of these two positions, the volunteers swallowed $15 \mathrm{ml}$ of half-strength liquid barium (Polibar, E-Z-E M, New York, NY) on command and had up to a maximum of 5 swallows per site. During each swallow, concurrent recordings of video fluoroscopy, velocimetry, and manometry were made. Radiation exposure was strictly monitored and limited to a total of $70 \mathrm{~s}$. Between barium swallows, volunteers drank water to clear the sensor and esophagus of residual contrasts.

Manometry. The recording assembly was a 23-lumen silicone rubber extrusion, with an external diameter of $4.2 \mathrm{~mm}$ (Dentsleeve, Wayville, SA, Australia). Two lumens were used to carry the transmitting and receiving optical fibers that led back up to the light source and fiber coupler, respectively ( $\mathrm{Fig}$. 2). A chain of 20 sideholes at $1.5-\mathrm{cm}$ intervals was used for recording pressures via 0.4-mm-diameter lumens. The laserDoppler sensor tip was installed $7.5 \mathrm{~cm}$ proximal to the most distal manometric sidehole. Manometric channels were each perfused at $0.15 \mathrm{ml} / \mathrm{min}$, giving pressure rise rates of at least $160 \mathrm{mmHg} / \mathrm{s}$. Data were acquired at $50 \mathrm{~Hz}$ in a customwritten program (HAD, G. S. Hebbard, Dept. of Gastrointestinal Medicine, Royal Adelaide Hospital) in Labview 3.1.1 (National Instruments) and were averaged to $10 \mathrm{~Hz}$, digitized, and logged on the master computer (Power Macintosh 7100/80).

Veloci metry. Velocimetry measurements were made at 4- 7 $\mathrm{Hz}$ and logged concurrently with the manometric recordings on the master computer.

Radiol ogy. All views were appropriately coned and shiel ded. The level of the laser sensor tip was rendered radiologically opaque by tantalum wires inserted within channels not being used for manometry at that level. A lateral oblique projection was used for swallows with the probe in the lower esophagus, which aimed to include the lower esophageal sphincter and diaphragmatic hiatus. When the sensor was in the upper esophagus, a slightly oblique posteroanterior projection was used, with the neck and thoracic inlet in view. The swallows were recorded directly to videotape at 30 frames/s. No still frames were used.

Synchronization. A timing device (TD-100-S, Provideo Systems, Adelaide, Australia) generated and simultaneously sent a number, at $10 \mathrm{~Hz}$, to both the video tape (fluoroscopy) and the master computer (which logged both manometry and velocimetry data). This numerical code was then visible on review of both the video and combined manometric and velocity data, enabling corresponding events to be correlated to within $0.1 \mathrm{~s}$.

Data definitions and analysis. The recordings of pressures and velocities were translated into AcqKnowledge (Biopac, Goleta, CA) for display and analysis. Video fluoroscopic images of the barium flow patterns were analyzed separately from the manometric and velocity data. Two observers each independently recorded the timing of 12 items for each swallow (4 from fluoroscopy, 5 from velocimetry, and 3 from manometry).

The fluoroscopic items scored were as follows: 1) initial upward movement of the velocimeter sensor on swallowing (taken as the swallow reference time), 2) first appearance of contrast at the level of the velocimeter sensor, 3) departure of the trailing edge of the column of barium from the vel ocimeter sensor, and 4) lumen occlusion at the level of the velocimeter sensor.

The items scored during velocimetry were the onsets of 1) the initial and 2) the major velocimeter signals associated with each swallow, 3) the duration of the major signal, 4) the peak vel ocity of the major signal, and 5) the number of signals associated with each swallow. By virtue of the signal processing on-line, the velocimeter signal in the absence of flow was steady at $0 \mathrm{~m} / \mathrm{s}$; deflections were thus easily recognized and were defined as a clear departure of the velocimeter signal from the baseline by $10 \mathrm{~m} / \mathrm{s}$ or more for $0.5 \mathrm{~s}$ or longer. The major signal was defined as the deflection during which the greatest vel ocity was measured by the velocimeter, and, if the same peak vel ocity was recorded in more than one deflection for a given swallow, the deflection with the longest duration was judged to be the major signal.

The items scored during manometry were 1) the onset of the swallow-induced pharyngeal pressure wave, 2) the onset of the esophageal body common cavity pressure rise that occurs with entry of liquid boluses into the esophagus before the onset of the peristaltic pressure wave, and 3) the onset of the major upstroke of the esophageal body peristaltic pressure wave at the level of the vel ocimeter sensor.

Mean velocities and numbers of deflections were compared using an unpaired Z-test for comparisons of means. Differences in proportions were assessed with a $2 \times 2$ contingency table and calculation of $\chi^{2} \mathrm{P}$ value. The level of statistical significance was taken to be at $P<0.05$

\section{RESULTS}

\section{Bench Testing}

Turntablevalidation. A linear relationship was found between the known velocity of liquid on the turntable and the measured velocity with thelaser-Doppler velocimeter for bidirectional fluid movements (Fig. 3).

Tissue safety assessment. No evidence of tissue damage or perforation could be identified macroscopically or on serial histological sections.

\section{Human Study}

The protocol was well tolerated by all subjects, and no adverse effects were detected. Fifty swallows were recorded with concurrent velocimetry, fluoroscopy, and manometry in the eight subjects, 24 with the sensor situated in the proximal esophagus and 26 with it in the distal esophagus. Each subject contributed between 5 and 10 swallows, from 2 to 5 with the sensor in the proximal and 3 to 5 with the sensor in the distal esophagus.

There was excellent interobserver agreement in the assignment of times to the fluoroscopic, velocimetric, and manometric variables, with $89 \%$ concurrence to within $\pm 0.2 \mathrm{~s}$ and $96 \%$ concurrence to within $\pm 0.4 \mathrm{~s}$.

F igure 4 shows the typical combined manometric and velocimeter recordings from swallows with the sensor in the two sites. The velocimeter gave a signal between the time of initiation of a swall ow and the time of lumen occlusion at the level of the sensor in 46 of the 50 swallows (92\% for both proximal and distal sensor sites). In the remaining four swallows (3 subjects), there was no vel ocimeter signal (2 each with the sensor in the proximal and distal esophagus). In two of these subjects, the velocimeter subsequently registered a signal after the subjects drank 50-200 ml of water. The third subject was extubated directly following the swallow, which did not register a velocimeter signal, as his X-ray exposure time did not allow further evalua- 


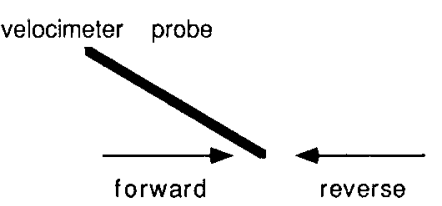

Test fluid velocity on turntable $\mathrm{mm} . \mathrm{s}^{-1}$

Recorded velocity from laser-Doppler velocimeter $\mathrm{mm} \cdot \mathrm{s}^{-1}$

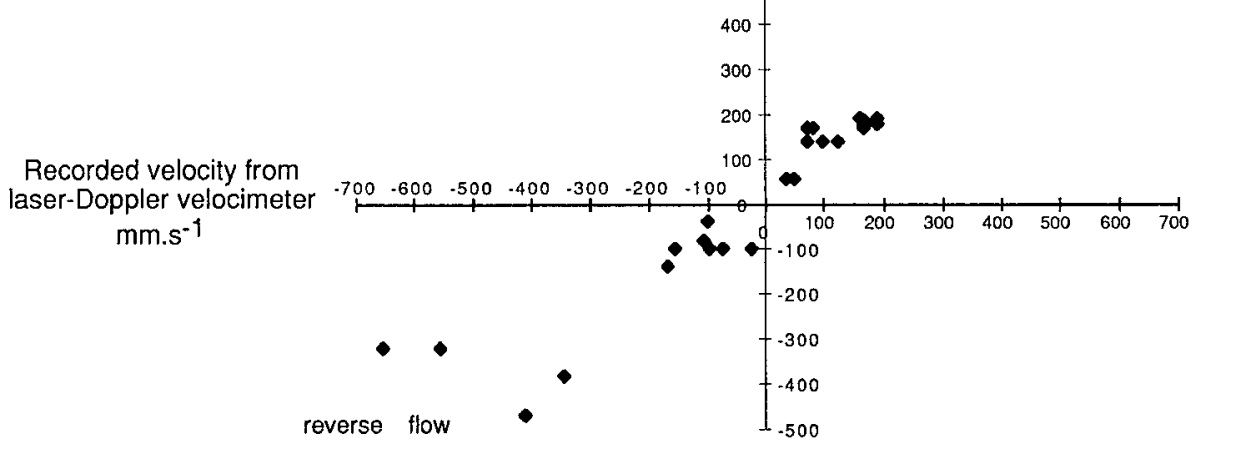

Fig. 3. Data from calibration of velocimeter against the grooved disk: velocimeter reading (x-axis) is plotted against actual velocity of fluid within the grooves (y-axis), which is being rotated at a controlled rate by the turntable. tion and the sensor was found to be heavily coated with barium.

The velocimeter signal had between 1 and 13 deflections during each swallow, with a greater number of deflections when the sensor was located in the distal compared with the proximal esophagus (4.3 vs. 2.4, P = 0.001 ). No velocimeter flow signal had its onset after fluoroscopic lumen occlusion or after the onset of the major upstroke of the peristaltic pressure wave at the level of the sensor. Maximal flow rates recorded with the vel ocimeter did not differ between the proximal and distal esophagus (76.7 vs. $73.8 \mathrm{~mm} / \mathrm{s}, \mathrm{P}=0.4$ ).

Compari sons between fluor oscopy and vel ocimetry. In half the swallows available for examination (22/44), the initial velocimeter signal commenced within $\pm 0.2 \mathrm{~s}$ of the upward movement of the assembly, before barium arrival at the sensor level (12 proximal, 10 distal). In the other half, the initial velocimeter signal occurred when contrast was at the level of the sensor (8 proximal, 14 distal). The relationship of the initial velocimeter signal to barium arrival at the sensor level could not be determined in two swallows because the fluoroscopic recording commenced after barium had arrived at the level of the sensor. Figure 5 gives a more detailed depiction of the temporal relationship between barium arrival at the level of the sensor and the initial velocimeter signal. The relationship between initial upward movement of the sensor and the initial velocimeter
A

Fig. 4. Concurrent pressures and velocimeter recordings during barium swallows, with the velocimeter sensor located in the proximal $(A)$ and distal (B) esophagus. Highest recording point shown is in the region of the upper esophageal sphincter. The manometric channel at the level of the velocimeter sensor is indicated by arrows. The dashed lines indicate the onset of the major upstroke of the peristaltic wave at the level of the sensor.

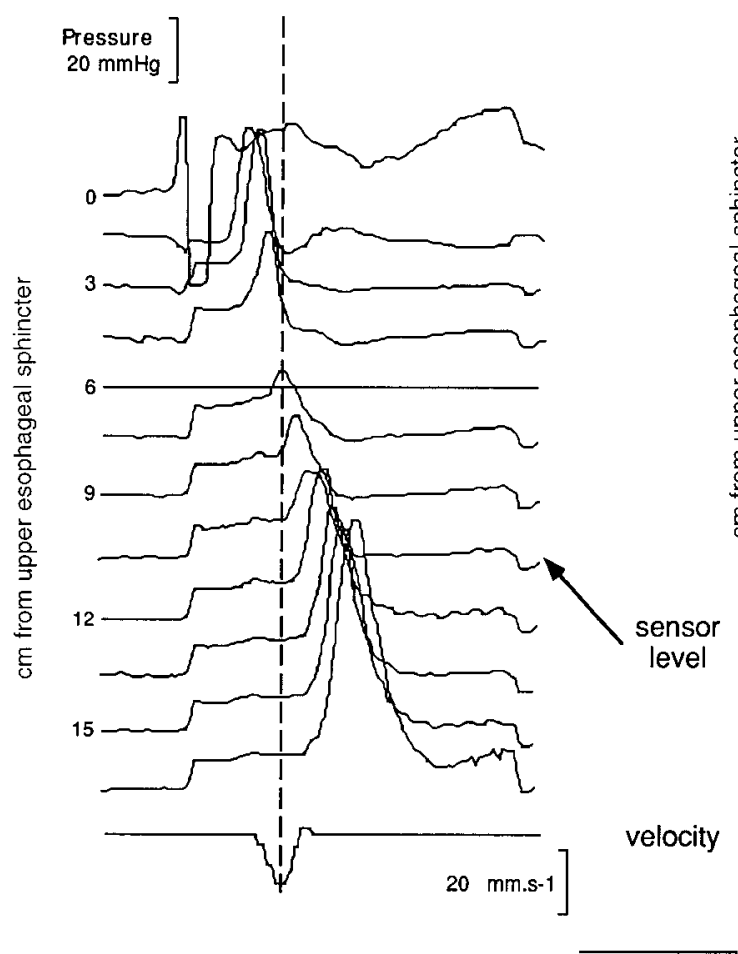

B

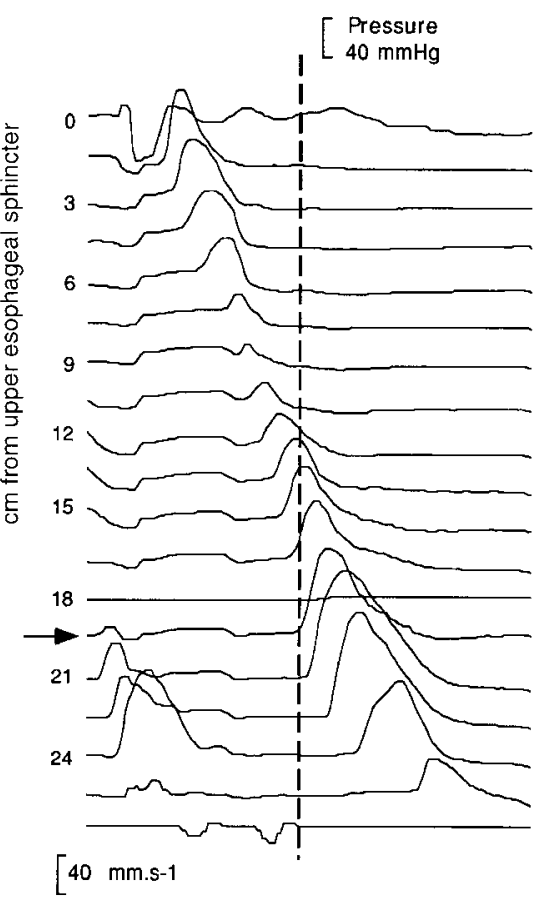


A
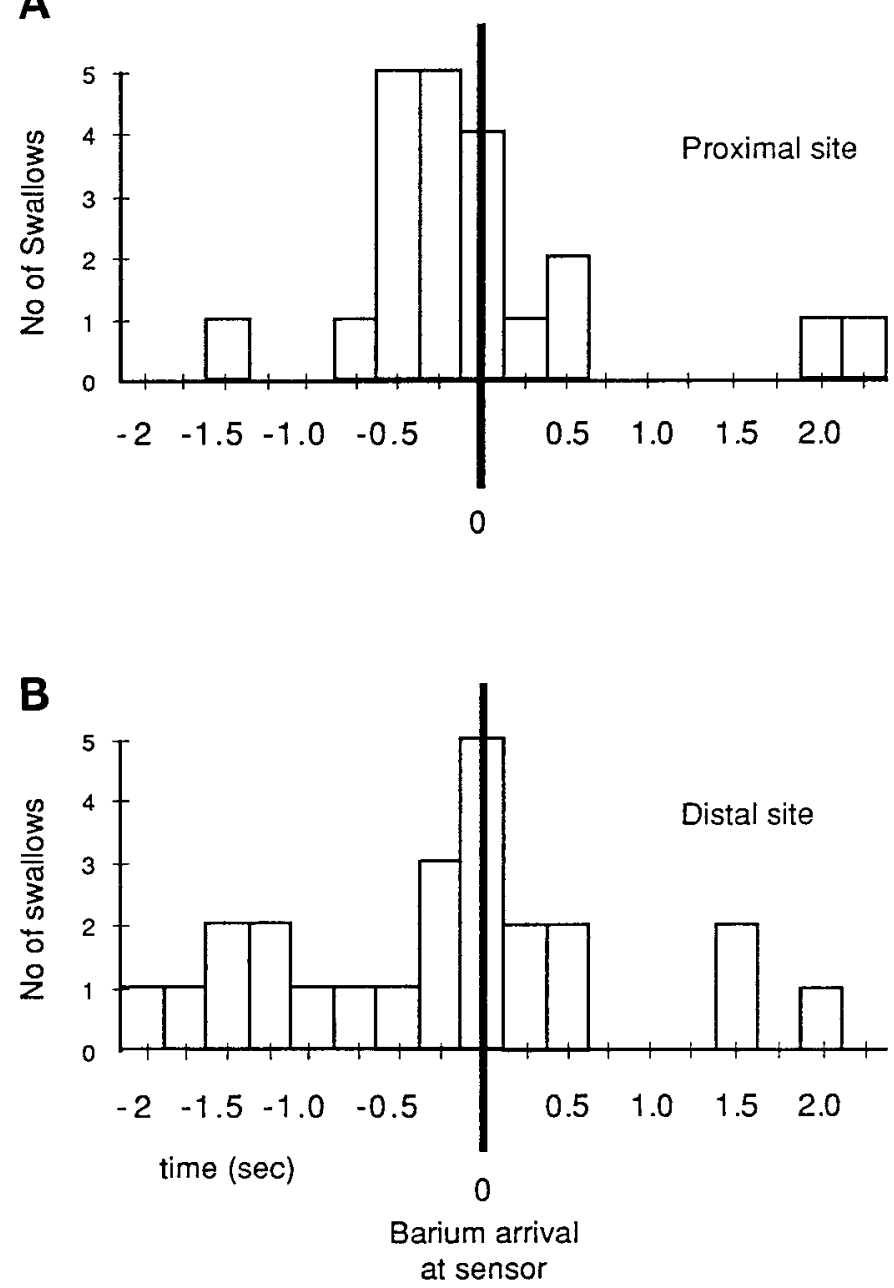

Fig. 5. Relationship between onset of the initial velocimeter signal and visible arrival of barium at the level of the sensor is shown separately for the swallows with the sensor in the proximal (A) and distal (B) esophagus. Bold vertical lines indicate the arrival time of barium. In the proximal esophagus, there is close temporal clustering of velocimeter signal onsets around the time of the onset of visible barium (17 of 21 swallows have the onset of the initial velocimeter signal within $\pm 0.5 \mathrm{~s}$ of the visible arrival of barium at the level of the sensor). This clustering is also seen with the sensor in the distal esophagus but is less impressive (13 of 24 swallows with initial signal and barium arrival within $\pm 0.5 \mathrm{~s}$ of each other). Eight swallows with the sensor in the distal esophagus had an initial velocimeter signal onset $>0.5 \mathrm{~s}$ before barium arrival. On fluoroscopic review of these swallows, the velocimeter signal is closely temporally related to upward catheter movement (see Fig. 6). In the 5 swallows (2 proximal, 3 distal) in which the initial velocimeter signal onset was $>0.5 \mathrm{~s}$ after barium arrival at the sensor, fluoroscopic review showed that visible barium was still present and lumen occlusion had not yet occurred.

signal is shown in Fig. 6. In the proximal esophagus, there was a greater proportion of swallows in which the timings of the initial velocimeter signal and barium arrival at thelevel of the sensor were closely temporally associated ( $\pm 0.5 \mathrm{~s})$ compared with the distal esophagus, although this did not quite reach statistical significance (81 vs. $54 \%, \mathrm{P}=0.057$ ).

In 21 of 46 swallows during which the velocimeter recorded a signal, the initial and the major velocimeter signals were identical. The major velocimeter signal commenced before barium reached the sensor in 13 swallows (10 proximal, 3 distal), while the sensor was within the column of barium in 28 swallows (9 proximal, 19 distal), or after the column had left the sensor (but traces remained on the mucosa) in 4 swallows (2 proximal, 2 distal). Figure 7 gives a detailed depiction of the temporal relationship between the major velocimeter signal and barium arrival at the level of the sensor. Figure 8 shows the relationship between the major velocimeter signal and the initial upward movement of the sensor on swallowing. The major vel ocimeter signal and barium arrival at the level of the sensor were more closely temporally associated ( $\pm 0.5 \mathrm{~s}$ ) for the proximal esophageal recordings compared with the distal esophagus ( 71 vs. $29 \%, P<0.005$ ). Swallows with the sensor situated in the distal esophagus were more likely to have the major velocimeter signal commencing more than $0.5 \mathrm{~s}$ after barium arrival at the level of the sensor compared with the proximal esophagus (71 vs. $24 \%$, $\mathrm{P}<0.002)$.
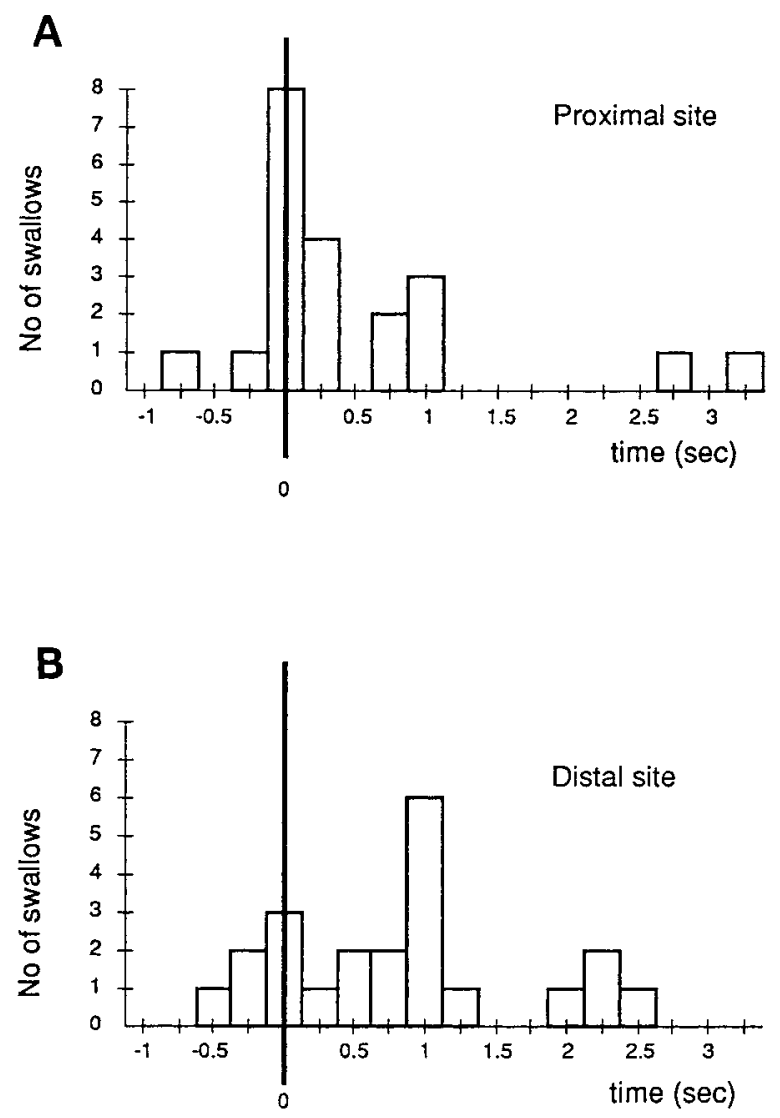

\section{Upward sensor movement}

Fig. 6. Relationship between onset of the initial velocimeter signal and initial upward movement of the sensor is shown separately for the swallows with the sensor in the proximal (A) and distal (B) esophagus. Bold vertical lines indicate the timing of the initial upward movement of the sensor. In the proximal esophagus, there is close temporal clustering of velocimeter signal onsets around the time of upward movement of the sensor (13 of 21 swallows within $\pm 0.5 \mathrm{~s})$. This relationship does not appear to hold true for the swallows with the sensor in the distal esophagus. 

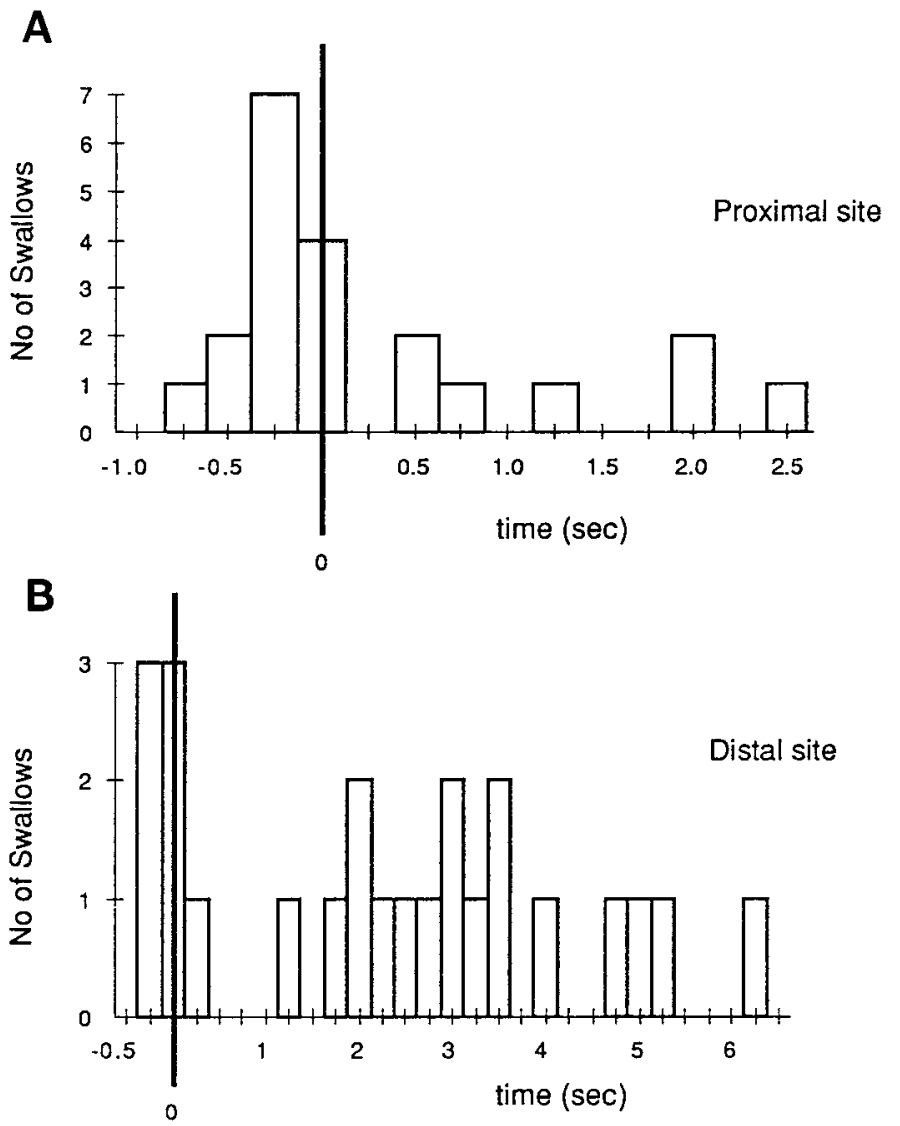

Fig. 7. Relationship between onset of the major velocimeter signal and visible arrival of barium at the level of the sensor is shown separately for the swallows with the sensor in the proximal $(A)$ and distal (B) esophagus. Bold vertical lines indicate the arrival time of barium. In the proximal esophagus, there is close temporal clustering of major velocimeter signal onsets around the time of barium arrival at the sensor ( 15 of 21 swallows have these 2 observations within $\pm 0.5 \mathrm{~s}$ ). I $\mathrm{n}$ the distal esophagus, this is seen to a far lesser extent ( 7 of 24 swallows within $\pm 0.5 \mathrm{~s}$ ), with the majority of the swallows (17 of 24 ) having the major vel ocimeter signal onset later. On review of the fluoroscopy in these swallows, maximal vel ocity was more frequently related to the emptying phase (as the barium column left the sensor) than the filling phase (as barium arrived at the sensor) of the bolus passage. In all instances, barium was still present and lumen occlusion had not yet occurred.

In the proximal esophagus, both the initial and the major velocimeter signals were cl osely temporally associated with the initial upward movement of the sensor and the arrival of barium at the level of the sensor (initial signal: $81 \%$ within $\pm 0.5 \mathrm{~s}$ of barium arrival and $62 \%$ within $\pm 0.5 \mathrm{~s}$ of upward movement; major signal: $71 \%$ within \pm 0.5 s of barium arrival and $67 \%$ within $\pm 0.5 \mathrm{~s}$ of upward movement), whereas in the distal esophagus neither the initial nor the major velocimeter signals appeared to be closely related to upward movement of the sensor $(41 \%$ of initial signals and only $8 \%$ major signals were within $\pm 0.5 \mathrm{~s}$ of upward movement). There was a shorter time interval between the initial upward movement of the sensor and the arrival of barium at thelevel of the sensor when the sensor was in the proximal compared with the distal esophagus [proximal mean of $0.5 \mathrm{~s}$ (range $0.2-0.9 \mathrm{~s}$ ) vs. distal mean of $1.0 \mathrm{~s}$ (range $0.4-2.5 \mathrm{~s}$ ), $\mathrm{P}<0.001$ ].
Comparisons between manometry and velocimetry. The initial velocimeter signal had an onset within \pm 0.5 $s$ of the onset of the esophageal common cavity pressure rise at the level of the velocimeter sensor in 28 of 46 swallows (16 proximal, 12 distal). The onset of the major velocimeter signal occurred during or within $\pm 0.5 \mathrm{~s}$ of the onset of the esophageal body common cavity pressure rise at the level of the velocimeter sensor in 36 of 46 swallows (16 proximal, 20 distal). No velocimeter signals had their onset after the onset of the major upstroke of the esophageal peristaltic pressure wave at the level of the sensor.

When the manometric recordings of esophageal body peristalsis were classified as normal $(n=27)$ or abnormal $(n=19)$ by established criteria (7), there was no difference in the number of velocimeter deflections between the normal (3.22 deflections) and the abnormal (3.58 deflections) swallows. Even when divided by sensor site, no difference in number of deflections was shown between the normal (2.62 proximal vs. 3.79 distal deflections) and abnormal (2.11 proximal vs. 4.9
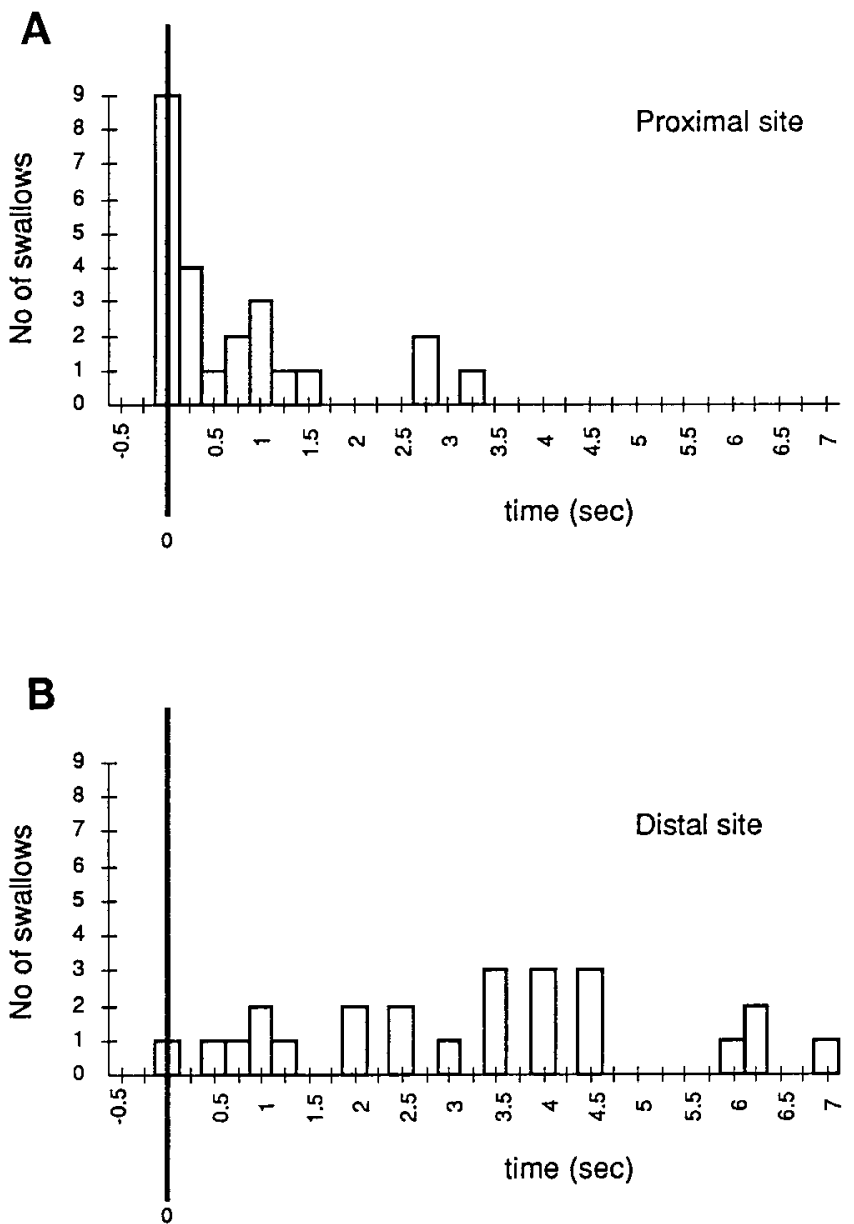

Fig. 8. Relationship between onset of the major velocimeter signal and initial upward movement of the sensor is shown separately for the swallows with the sensor in the proximal (A) and distal (B) esophagus. Bold vertical lines show the timing of the initial upward movement of the sensor. In the proximal esophagus, the majority of the major velocimeter signals have their onsets within the $1.5 \mathrm{~s}$ following upward movement of the sensor. In the distal esophagus, upward catheter movement appears to have no effect on the timing of the major velocimeter signal. 
distal deflections) swallows ( $\mathrm{P}>0.05$ for all). There was also no difference in the maximal velocity recorded during manometrically normal $(69 \mathrm{~mm} / \mathrm{s}$ for proximal, $55.9 \mathrm{~mm} / \mathrm{s}$ for distal) and abnormal $(80.1 \mathrm{~mm} / \mathrm{s}$ for proximal, $63.4 \mathrm{~mm} / \mathrm{s}$ for distal) swallows $(\mathrm{P}>0.05$ for both).

\section{DISCUSSION}

The main aim in developing the instrument de scribed in this report was to be able to recognize individual episodes of luminal flow in the human gut, a capability that is not adequately provided by any established method. The development of a more complete understanding of the relationships between flow pulses and spatiotemporal patterns of intraluminal pressures depends on being able to recognize and monitor luminal flow episodes with high time resolution. In the present study, this laser-Doppler device has been shown to be safe and well tolerated and to be able to accurately detect flow with a temporal resolution of $<1 \mathrm{~s}$. The in vivo study provides strong direct evidence of the ability of the device to recognize intraluminal flow episodes under expected conditions of use, with a high detection rate (92\%) for barium flow in the esophagus compared with fluoroscopy, and, moreover, differences in flow patterns between the proximal and distal esophagus that were consistent with those seen on fluoroscopy were detected.

For the initial testing in humans of the laser-Doppler vel ocimeter, we chose to use the esophagus because we could reliably trigger and image movements of luminal contents fluoroscopically within acceptable radiation limits. Along with its ability to detect flow, the velocimeter also revealed apparent differences in flow patterns between the proximal and distal esophagus. In particular, the flow pattern signaled by the vel ocimeter is more simple in the proximal esophagus, with fewer flow pulses (deflections) per swallow. Fluoroscopic observation of the barium movement showed good agreement with the velocimeter recording, with straight-forward, unidirectional flow in the proximal esophagus occurring soon after swallowing. Our observations of barium flow in the distal esophagus are consistent with those of Biancani and Behar (2) who describe liquid flow occurring in two distinct phases: 1) during filling against a closed lower esophageal sphincter and 2) during emptying of the esophagus after the opening of the sphincter. Moreover, there is a variable time interval, from swallow to swallow (and between subjects), between the filling and emptying flow phases in the distal esophagus, during which to and fro movement of the column of barium can sometimes be seen. These fluoroscopically observed flow patterns in the distal esophagus are likely to account for the greater number of velocimeter signal deflections that occurred over a longer time interval in the distal esophagus.

In a number of swallows (22 of 44), the onset of the initial velocimeter signal appeared to precede fluoroscopic flow, particularly when the sensor was in the proximal esophagus. On fluoroscopy, these "premature" flow signals were closely related to the rapid upward movement of the recording assembly on swallowing, although timing uncertainty (see below) precludes a definite conclusion. Initial, rapid upward movement (shortening) of the pharynx and esophagus occurs on swallowing (6), and, based on our observations, rapid upward movement of the recording assembly also occurs, although these two upward movements (of the probe and the esophagus) are not necessarily synchronous or equal, as the assembly is not fixed to the esophagus. Because the velocimeter simply records movement of particles relative to the sensor, movement of either the recording assembly or the mucosa or an unequal movement between them will generate a signal. The vel ocity of the upward movement of the sensor seen fluor oscopically is certainly sufficient to fall within the resolution of measurement of the velocimeter $(>30$ $\mathrm{mm} / \mathrm{s}$ ). Formal evaluation under standardized conditions is warranted to clarify the issue of signals arising from relative movement between sensor and mucosa.

The differences between the two methods used to sense flow in this study are important, as they have influenced the data obtained. Fluoroscopy gives an indication of volumes flowing and the movement of the leading and trailing edges of a bolus, whereas the vel ocimeter gives information on timing and velocity of flow in the immediate region of the sensor but does not signal volume. Fluoroscopy of barium swallows, by outlining the mucosa, may also allow lumen occlusion to be visualized if sufficient air contrast is present. Fluoroscopy is limited by the need for visual interpretation and the density of barium used. In this study, the barium used was half strength (to reduce sensor clogging), increasing the likelihood that the temporal resolution of the fluoroscopy was decreased. In contrast, interpretation of thelaser-Doppler velocimeter's recording is less subjective. When these differences are considered, the substantial concurrence of flow detected by the two methods in this study strongly supports the velocimeter's performance.

The analysis of our in vivo data required somewhat subjective definitions of events. To counter this, the process was highly structured, with two observers independently evaluating the data sets. The high concordance between the analyses of the two observers strongly supports the validity of this approach.

We found no overall difference between manometrically "normal" and "abnormal" swallows in maximum velocity or number of flow episodes recorded by the vel ocimeter, although there was a wide range of vel ocities and flow patterns recorded, attesting to the fact that flow patterns vary from one swallow to the next and between individuals. It was somewhat surprising that maximal flow velocities, as measured by the velocimeter, were no different between proximal and distal sites; when the passage of contrast radiologically was viewed, the rate of advance of the bolus front appeared to be faster in the proximal esophagus. It is likely that the shorter time from swallow to contrast appearance at the sensor level $(0.2-0.9 \mathrm{~s}$ proximally vs. 0.4-2.5 s distally) influenced our visual judgment of this. Thelack of difference in vel ocities between the two sites may also partially relate to the differences be- 
tween fluoroscopic and velocimetric measurement of flow (see above).

From our data, by both velocimetric and fluoroscopic assessment, esophageal luminal flow was largely completed before the arrival of the peristaltic pressure wave at any given point. This is consistent with previous observations of liquid flow in the upright position (2), as gravity accounts for a large component of the flow. The spatiotemporal patterning of pressures in the esophagus is, however, important in transport of solids regardless of posture. The causal relationship between pressures and flows is likely to be better understood when regions in which gravity plays a lesser role are evaluated, such as in the pylorus and proximal small intestine. At the time of lumen occlusion judged fluoroscopically, some fluid movement was still being signaled by the velocimeter, although no velocimeter signals commenced after this time. This is likely because of flow in the thin layer of liquid left coating the mucosa as it is squeezed during lumen occlusion; if so, this is real flow, although of a small volume, emphasizing the different nature of flow monitoring between the two techniques as discussed earlier.

The characteristics of the instrumentation used unfortunately resulted in some timing uncertainty. The manometric data were recorded to disk at $10 \mathrm{~Hz}$, while the vel ocimeter gave measurements at $4-7 \mathrm{~Hz}$ due to limitations of the components processing the signal. Consequently, there was an unavoidable mismatch of the two signals that reduced the temporal resolution. In addition, the internal processing of the velocimeter signal, from measurement of the Doppler shift to logging with the manometry, created a time lag between the vel ocimeter signal and the manometric data. Compensation for this time delay was complicated by the fact that it was not constant but varied slightly from one swall ow to the next (between $\sim 0.3$ and $0.6 \mathrm{~s}$ ). Because it was not possible to retrospectively define this delay precisely for each swallow, an average delay of $0.4 \mathrm{~s}$ was taken from 10 standardized (video re corded) movements performed in vitro before analysis of the data. This figure was then used as a best estimate to correct for these uncertainties in temporal resolution between the velocimetric and manometric recordings. From these considerations, events within $\pm 0.2 \mathrm{~s}$ of each other may in fact be simultaneous. This uncertainty in timing can only be resolved by modifying the system so that the velocimeter measures at the same frequency as the manometric device $(10 \mathrm{~Hz})$ and eliminating or standardizing the internal delay in signal processing. These technical aspects are currently being addressed in further development of the instrument.

If the box containing the spectrum analyzer was bumped or exposed to radio frequency-emitting devices, the vel ocimeter system used in this study was noted to give signals when no flow was occurring at the sensor. These artifactual signals demanded special care to be taken during use of the device. This limitation could be addressed by measures that will increase stability and shielding from external electromagnetic signals. The clogging of the sensor with barium is more of a nuisance than a serious technical problem; use of other contrast strategies during instrument development could help to avoid this problem. When the velocimeter is sufficiently validated to be used alone, a number of other solutions that are less likely to coat the sensor, such as dilute milk and lipid emulsions, are suitable alternatives, as the only absolute requirement is the presence of particles in the liquid to reflect the laser beam.

The novel instrument described here is capable of making a significant contribution to an improved understanding of human gastrointestinal mechanics; it is safe, well tolerated, and performs intraluminal flow measurements with a temporal resolution of better than $1 \mathrm{~s}$. The initial experience described in this report indicates the need for enhancement of some aspects of its function. Once these enhancements have been achieved, the vel ocimeter may enable us to monitor and thus evaluate pressure-flow relationships in the human upper gut.

This work was supported by project grant funds from the National Health and Medical Research Council (NHMRC) of Australia, the Special Purposes Fund at the Royal Adelaide Hospital, and Astra (Sweden). J. M. Andrews was in receipt of an NHMRC Medical Postgraduate Scholarship. C. K. Rayner was in receipt of a Dawes Scholarship from the Royal Adelaide Hospital .

Address for reprint requests and other correspondence: J . M. Andrews, Dept. of Medicine, Royal Adelaide Hospital, North Terrace, Adelaide, SA 5000, Australia (E-mail: jandrews@medicine.adelaide.edu.au).

Received 25 August 1998; accepted in final form 5 December 1998.

\section{REFERENCES}

1. Berstad,A., T. Hausken, O. H. Gilja, N. Thune, K. Matre, and S. Odegaard. Volume measurements of gastric anrum by 3-D ultrasonography and flow measurements through the pylorus by duplex technique. Dig. Dis. Sci. 39, Suppl. 12: 97S-100S, 1994.

2. Biancani, P., and J . Behar. Esophageal motor function. In: Textbook of Gastroenterol ogy, edited by T. Yamada. Philadel phia, PA: Lippincott, 1995, p. 158- 180.

3. Cannon, W. B. The movements of the intestines studied by means of the roentgen rays. Am. J . Physiol. 6: 251-277, 1902.

4. Feldman, M., H. J . Smith, and T. R. Simon. Gastric emptying of solid radiopaque markers: studies in healthy subjects and diabetic patients. Gastroenterol ogy 87: 895-902, 1984.

5. Foulk, W. T., C. F. Code, C. G. Mortlock, and J . A. Bargen. A study of the motility patterns and the basic rhythm in the duodenum and the upper part of the jejunum in human beings. Gastroenterol ogy 26: 601-611, 1954.

6. Kahrilas, P.J ., W. J . Dodds, J . Dent, J . A. Logerman, and R. Shaker. U pper esophageal function during deglutition. Gastroenterology 95: 52-62, 1988.

7. Kahrilas, P. J., W. J. Dodds, and W. J . Hogan. Effect of peristaltic dysfunction on oesophageal volume clearance. Gastroenterol ogy 94: 73-80, 1988.

8. Malbert, C. H., A. Latour, C. Dardillat, and Y. Ruckebusch. Measurement of gastric emptying rate. J. Biomed. Eng. 9: 180-182, 1987.

9. Malbert, C. H., and Y. Ruckebusch. Relationships between pressure flow across the gastroduodenal junction dogs. Am. J. Physiol. 260 (Gastrointest. Liver Physiol. 23): G653-G657, 1991.

10. Nathan, H., S. Macdonald, J . M. Andrews, C. H. Malbert, D. Kilpatrick, J. Dent, C Frisby, and E. Staunton. A laser Doppler velocimeter for the measurement on gastric flows (Abstract). Neurogastroenterol. Motil. 10: A126, 1998.

11. Parkman, H. P., M. A. Miller, R. S. Fisher. Role of nuclear medicine in evaluating patients with suspected gastrointestinal motility disorders. Semin. Nucl. Med. 25: 289- 305, 1995.

12. Silny, J . Intraluminal multiple electric impedance procedure for measurement of gastrointestinal motility. J . Gastrointest. Motil. 3: 151-162, 1991.

13. Standards Australia. Laser Safety (AS2211). North Sydney: Standards Assoc. Australia, 1991. ISBN 0726270105. 